\title{
Phenotypic evaluation of DH-lines developed on the base of rice samples with blast resistance genes
}

\author{
E. G. Savenko*, Zh. M. Mukhina, V. A. Glazyrina, T. L. Korotenko, and S. V. Garkusha \\ Federal Scientific Rice Centre, Krasnodar, Russia
}

\begin{abstract}
Gametoclonal changes are an additional source of genetic variation suitable for use in crop improvement programs for a range of agronomic traits. The aim of the study was to accelerate development of genetically stable DH rice lines with high morphological and genetic uniformity by the method of experimental haploidy in vitro based on breeding valuable samples with specified characteristics and carrying genes of a wide spectrum of blast resistance, as well as samples with racespecific genes for resistance to the pathogen, and subsequent assessment of agronomic traits of androgenic lines. The data on the callusogenic and regenerative capacity of 30 genotypes of domestic rice and samples of Chinese introduction with genes for blast resistance in anther culture in vitro are presented. Genetically stable (homozygous) androgenic lines were developed on the basis of these breeding valuable samples. The characteristics of economic and biological traits and elements of panicle productivity of $30 \mathrm{DH}$-line developed from three samples with blast resistance genes are given. The variability of traits arising as a result of gametoclonal variability in androgenic lines was noted for such traits as plant height, panicle length, blast resistance, and weight of 1000 grains.
\end{abstract}

\section{Introduction}

Current estimates indicate that the world's population will surpass nine billion by 2050 . The issues of developing new varieties and hybrids of agricultural crops are a kind of "locomotive" of food security of each state. The work of breeding centers is coming to the forefront of the agro-industrial complex, which face a serious task - development of radically new drought-resistant, disease-resistant and high-yielding varieties and hybrids of the main agricultural crops. Rice is one of the three most cultivated cereals, which is the basis of the nutrition of millions of people around the world, therefore the development of the rice industry, mainly breeding, is imperative. The genetic basis for the implementation of breeding programs in various directions is provided by pre-breeding work to develop sources and donors of breeding valuable traits. Development of varieties through hybridization takes both time and resources. To breed lines with an acceptable level of homozygosity before testing them for agronomic parameters, at least six generations are

\footnotetext{
* Corresponding author: avena5@ rambler.ru
} 
required. From the moment of crossing, until phenotypically homogeneous lines are obtained, it takes from 8 to 10 years. They are then evaluated for 3 years or more to identify potential candidate lines for varieties. The constant demand for new varieties with specific characteristics requires modern plant breeding methods to accelerate the development of new varieties. [1]. The efficiency of plant germplasm development can be increased by developing genetically stable samples using modern biotechnology methods. Genetic stabilization of breeding material using experimental haploidy (isolated anther culture in vitro) is an important component of the breeding scheme. Doubled haploids have numerous advantages: the diversity and homogeneity of the developed lines; high efficiency of selection of target genotypes, because the action of recessive genes manifests itself along with dominant ones, therefore, in their population, there is a wide range of variation in traits controlled by recessive genes; dihaploids with genes that determine productivity, disease resistance, biotic and abiotic factors are of great interest for practical breeding purposes [2-5]. The main steps associated with the production and use of DH technology include haploid induction (in vitro or in vivo), identification of haploid seeds (using morphological or DNA markers), duplication of haploid chromosomes, and finally generation of DH seeds to obtain DHL [5].

The production of doubled haploids is limited by many factors affecting the efficiency of cultivation, such as the genotype of the explant, the growing conditions of donor plants, the stage of microspore development, pretreatment of panicles [6], cultivation methods, media and cultivation conditions [7,8]. One of the main obstacles to the use of rice anther culture is the identification of microspores that respond to callus formation. For the successful production of androgenic haploids, it is important to establish a critical period in the development of a microspore, characterized by certain physiological and biochemical events, when it is possible to switch its development to the path of androgenesis. To do this, before introducing into in vitro culture, it is necessary to carry out a cytological assessment of the state of microspores and compare the relationship of their development with the morphological traits of the panicle [9]. Currently, there are reports on the use of morphological markers such as panicle length, spikelet location and color, anther color and its position in the spikelet for subsequent callus induction. Based on research by S.T. Mercy, F.J. Zapata (1986), the distance between the flag leaf and the bottom leaf, as well as the late mononuclear and early binuclear pollen stages can be used as markers for callus induction.

The technique for obtaining doubled haploids consists in growing calli or cell suspension cultures, followed by organ / embryogenesis and plant regeneration.

Regenerated plants and their progeny are evaluated in order to identify individuals with a new phenotype, because cell and tissue culture conditions cause a variety of genetic changes. Variations observed among plants regenerated from cultured gamete cells are called gametoclonal variations or gametoclonal variability (Evans et al., 1984) $[10,11]$. The mechanisms underlying genetic variation include: changes in the level of ploidy, changes in chromosome structure, gene mutations, gene amplification, changes in extra-nuclear genes, activation of transposed elements, and DNA methylation. Gametoclonal changes are an additional source of genetic variation suitable for use in crop improvement programs for a number of agronomic traits such as disease resistance, plant height, shoot number and yield, and various biochemical traits. The selection efficiency in the lines of doubled haploids $(\mathrm{DH})$ is higher, especially with significant variations in dominance [12]. Thus, variations in several agronomic traits were noted in DH wheat (Snape et al., 1988). Variations in traits in teff (Eragrostis teff (Zuccagni)), an important grain crop of Ethiopia obtained using gamete cells (gynogenesis), have been found for plant height, panicle length, seed size and ripening time (Gugsa and Loerz, 2013, Gugsa et al., 2006), and dihaploid potato plants obtained from anthers were genetically different from tetraploid forms, as well as anther donor plants 
due to gametoclonal variability that arose during androgenic dihaploidization (Sarkar et al., 2010). Several DH regenerated rice lines obtained through anther / microspore culture have shown significant variability in traits that can improve agronomic performance (Ying et al., 1996, Yoshida et al., 1998). Currently, rice gametoclones with disease resistance have been identified. Heszky and Simon-Kiss (1992) tested several gametoclonal variants originating from rice anther culture, one of which was released as Dama, Piricularia resistant and possessing good culinary quality.

Biotechnological and molecular analysis showed a high degree of genetic stability of gametoclones and their progeny (Dutta, 2005). Thus, anther culture can, to a certain extent, alter the characteristics of plants derived from gamete cells, influencing their use in plant breeding and genetic engineering programs [13].

The aim of the research is to accelerate the development of genetically stable androgenic lines with high morphological and genetic uniformity by the method of experimental in vitro haploidy based on breeding valuable samples with specified characteristics and carrying genes of a wide spectrum of resistance to blast, as well as samples with racespecific genes for resistance to the pathogen, assessment and phenotyping of polymorphism of quantitative traits of DH-lines developed by the anther culture method to increase the productivity of modern rice varieties.

\section{Material and methods}

Before the introduction into anther culture in vitro, a cytological assessment of the state of microspores was carried out and the relationship of their development with the morphological traits of the panicle was compared. Anthers for cytological control were taken in the period from the beginning of the emergence of the flag from the tube until the appearance of the upper spikelets. The stage of development of microspores was determined using fluorescence microscopy with staining gamete cells (microspores) with DAPI dye (4,6-diamidino-2-phenylindole dihydrochloride) (Sayfitdinova A.F., 2011). Cells were isolated from anthers ranging in size from 2.0 to $3.5 \mathrm{~mm}$. The length of the anthers was determined using an eyepiece with a scale of a stereomicroscope (binocular). Microspores under a binocular microscope were squeezed out of the anthers onto a glass slide using dissecting needles, adding a fixative dropwise as it evaporated (3 parts of $96 \%$ ethanol and 1 part of glacial acetic acid) for 10 minutes, stained with DAPI fluorescent dye for 12 minutes, then covered with a cover glass and microscoped in ultraviolet light (magnification 630 and 1000x). The developmental stage was determined by the number of nuclei and their location in the cell. In the rapid determination of the stage of development, rice microspores were squeezed out of the anthers onto a glass slide under a stereomicroscope, a drop of acetocarmine was added, covered with a cover glass and microscoped at 200 and 400 times magnification. The stage of development of microspores in the express method was determined by the form of microspores.

In the spring-summer period, donor plants were grown in a growing site and in the field, in the autumn-winter period in controlled conditions of artificial climate chambers at 18 $20^{\circ} \mathrm{C}$, relative humidity $70 \%$. The selection of the biomaterial of the material (immature panicles) was carried out in the morning. When studying the influence of the growing conditions of donor plants, it was found that the induction of callusogenesis from the anthers of plants grown in artificial climate chambers is lower than that of plants of the same genotype grown on a summer growing site or in field conditions due to different physiological characteristics of donor plants.

To stimulate the transition of most microspores to the mononuclear stage and increase their lifespan due to the formation of specific proteins of temperature shock, an increase in the frequency of callus formation, proliferation with the formation of morphogenic 
structures, panicles with anthers were preliminarily kept at a low positive temperature of 8$10^{\circ} \mathrm{C}$ for ten to fourteen days. Due to low positive temperatures, the destruction of the anther wall was delayed, which has a harmful effect on the development of microspores in culture. This technique also made it possible to preserve cut panicles for a long time, which is important in the mass cultivation of anthers.

Before planting the anthers on artificial nutrient media, the panicles were sterilized with a commercial solution "Belizna" for 10 minutes, washed three times in sterile distilled water. To reduce the risk of contamination, sterility rules developed for cell and tissue culture were followed. All manipulations were performed under aseptic conditions in a laminar box. The culture media were autoclaved at 1.2 atmospheres for 20-25 minutes.

To stimulate cell determination and induction of callusogenesis, Blaydes' medium (Blaydes, 1966) was used with 2,4-D (2,4-dichlorophenoxyacetic acid) at a concentration of $2.0 \mathrm{mg} / \mathrm{L}$, as a stimulating induction of callusogenesis in most genotypes and influencing the subsequent regeneration of plants from callus cells. The quality and quantity of calli were recorded for each sample. 150 anthers of each genotype were inoculated in triplicate. Anthers were cultivated in a thermostat at a temperature of $25 \pm 2{ }^{\circ} \mathrm{C}$, relative humidity of $50 \%$ in the dark for 20-30 days until the formation of calli. Callus aggregates were transferred to a nutrient medium Murashige and Scoogy (MS, 1962). The media included macro- and microsalts, Fe-chelate, organic additives (vitamins and amino acids), agar-agar and sugars. Calli were cultivated with a photoperiod of 12 hours - day (5000 lux), 12 hours - night until the emergence of seedlings, which were rooted on a hormone-free MS medium.

When performing genotyping, multiplex PCR analysis was used. To perform DNA analysis, an ABIprism 3130 automatic genetic analyzer was used, which makes it possible to identify the difference in the size of the amplified fragments with an accuracy of one base pair.

The establishment of experiments, counting, observation and phenotyping of the material was carried out according to the "Methodology of experimental work on breeding, seed production, seed studies and control over the quality of rice seeds" (A.P. Smetanin, V.A. Dzyuba, 1972).

\section{Results}

In order to obtain morphogenic callus lines and to accelerate development of genetically stable (homozygous) DH lines with high morphological and genetic uniformity by the method of experimental haploidy, anthers of 30 genotypes of domestic rice with genes of resistance to blast $\mathrm{Pi}-\mathrm{b}, \mathrm{Pi}-$ were introduced into in vitro culture: $\mathrm{Pi}-\mathrm{b}, \mathrm{Pi}-\mathrm{ta}, \mathrm{Pi}-1, \mathrm{Pi}-2, \mathrm{Pi}-$ 33, Pi-z, Pi-ks Pi-a, Pi-19, Pi-lm, Pi-m, Pi-30, Pi-ta 2, Pi-62 ( samples stored at USU "Collection of genetic resources of rice, vegetables and melons" All-Russian Rice Research Institute) and samples of Chinese introduction with target genes of resistance to blast Pigy8, Pita and Ptr (table 1).

The stage of development of microspores was determined by the express method according to the shape of microspores (Figure 1).

It was found that in rice, the mononuclear and early binuclear stages of microspore development are optimal for the formation of callus, thus, a correlation was revealed between the development of pollen and the position of the rice panicle during the booting period, which made it possible to visually record the optimal stage of selection of explants (anthers) by morphological traits, without resorting to cytological control. Based on the results of cytological studies, it was determined that for inoculation of anthers, panicles located in the sheath of the leaf should be selected, while the distance between the flag and the second leaf in different genotypes is $5-10 \mathrm{~cm}$ (2-3 days before sprouting); flower scales 
are light green; the length of the anthers is $2.0-2.5 \mathrm{~mm}$ (Figure 2). Thus, the distance between the flag and bottom leaf was used as a marker for selection of panicles.

Table 1. Samples with blast resistance genes for development of DH-lines.

\begin{tabular}{|c|c|c|c|}
\hline № & $\begin{array}{c}\text { Catalogue } \\
\text { number }\end{array}$ & Sample name & Gene presence \\
\hline 1. & 04072 & Snezhinka & Pi-b, Pi-ta \\
\hline 2. & 02890 & Mutant $744-82$ & Pi-ta \\
\hline 3. & 0590 & no name & Pi-zt \\
\hline 4. & 01717 & no name & Pi-z \\
\hline 5. & 04437 & L.9 & Pi-1, Pi-2, Pi-33 \\
\hline 6. & 04636 & $\mathrm{Lm}-3$ & Pi-1, Pi-33 \\
\hline 7. & 04434 & B 33-38,7 & Pi-1, Pi-33 \\
\hline 8. & 04438 & L.5 & Pi-1, Pi-2, Pi-33 \\
\hline 9. & 04433 & B $33-38,6$ & Pi-b, Pi-z \\
\hline 10. & 04435 & L.6 & Pi-1, Pi-2, Pi-33 \\
\hline 11. & $98-4$ & Furunishiri & $\mathrm{Pi}-\mathrm{z}$ \\
\hline 12. & 04-89 & Shin 2 & Pi-ks \\
\hline 13. & $70-05$ & Lemont & Pi-lm \\
\hline 14. & $265-08$ & JR BL 20-JR 24 & $\mathrm{Pi}-20$ \\
\hline 15. & 111-09 & № 22 & resistant to $\mathrm{Pi}$ \\
\hline 16. & 114-09 & Cuaro & disease resistant \\
\hline 17. & 208-09 & PSB Rc 94 & resistant to $\mathrm{Pi}$ \\
\hline 18. & $58-12$ & Aogugzhan & resistant to $\mathrm{Pi}$ \\
\hline 19. & $1-14$ & Sha-tiao-tsao & Pi-ks \\
\hline 20. & $2-14$ & Jshikari-Shiroke & Pi-i \\
\hline 21. & $3-14$ & Zenit & Pi-a, Pi-z \\
\hline 22. & 8-14 & Fukunishiki & $\mathrm{Pi-z}$ \\
\hline 23. & $11-14$ & Aichi-asachi & Pi-a, Pi-19 \\
\hline 24. & $25-14$ & JR-64 & Pi-30, Pi-ta 2, Pi-33 \\
\hline 25. & $68-14$ & Yashiri-mochi & $\begin{array}{l}\text { resistant to Pi (Pi-ta, } \\
\text { Pi-ta2, Pi-62) }\end{array}$ \\
\hline 26. & 71-14 M & Anamihikari & Pi-m \\
\hline 27. & $52-16$ & Sakha 105 & resistant to $\mathrm{Pi}$ \\
\hline 28. & China & Liaojing 168 & Pigy8, Pita, Ptr \\
\hline 29. & China & Liaoxing 21 & Pita, Ptr \\
\hline 30. & China & HY 11 & Pigy8, Pita, Ptr \\
\hline
\end{tabular}
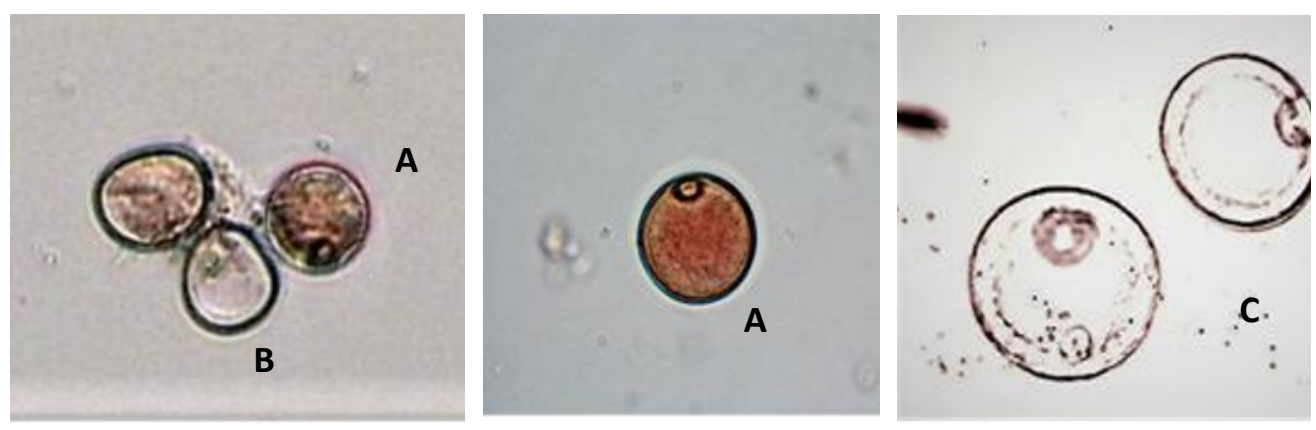

Fig. 1. Embryogenic microspores of rice at the late unicellular and early bicellular stages (A); nonembryogenic (B) (staining with acetocarmine, bright field, 1100x); embryogenic rice microspores without staining (C) (rapid determination). 


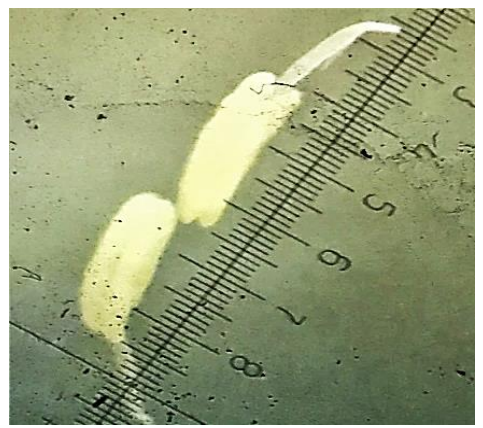

Fig. 2. Rice anther before introduction into in vitro culture.

Microspores in the anther of such rice panicles are capable, under in vitro conditions, of switching from the gametophytic developmental pathway to an abnormal development, as a result of which callus is induced, in which foci of meristem cells or callus embryoids are subsequently formed, followed by development into androgenic plants.

Analysis of the results showed that the induction of callusogenesis and regeneration was noted not in all studied rice genotypes. The intensity of the processes of callus formation and plant regeneration of these rice samples was largely due to genetic factors (Tables 1,2).

The induction of callusogenesis on a medium containing auxin 2,4-D at a concentration of $2.0 \mathrm{mg} / 1$ occurred on the 20th - 40th day, and was observed in most of the studied rice genotypes. The quality and quantity of calli were recorded for each sample. The variability in callus formation was genetically determined. As can be seen from Table 2, the indices of callusogenesis differed between the studied samples; they also varied between different plants - donors of the same sample.

When analyzing the parameters of callusogenesis, the maximum result was observed in sample No. $04072(26.31 \%)$, the minimum - 0.13\% in sample No. 58-12 (Table 2).

The best results for the realization of form-forming potencies and plant regeneration from calli of most of the studied genotypes were achieved on the Murashige and Skoog nutrient medium with $1.0 \mathrm{mg} / 1$ of $\alpha$-NAA and $5.0 \mathrm{mg} / 1$ of kinetin. The analysis of the experiments showed a wide range of fluctuations in the potential morphogenetic capabilities of the studied varieties and hybrids. The frequency of the formation of morphogenic structures and regeneration of green plants varied from 0 to $8.12 \%$. The variability in the regeneration trait largely depended on the genotype. Table 3 shows the minimum and maximum rates of regeneration from calli of different plants of the same sample.

The processes of morphogenesis began 10-20 days after the transfer of callus tissues to regenerating media and cultivation in the light. The maximum values for this trait were noted in samples No. 04434 and 04072 (8.12 and 7.82\%, respectively). The rest of the genotypes were characterized either by a lower frequency of the emergence of morphogenic structures and green seedlings, or by a complete absence of regenerative potencies. The age and morphology of calli directly influenced the processes of seedling regeneration. The highest regenerative capacity was possessed by a 20-30-day-old dense, white, fine-grained nodular callus of a white or light yellow shade with white blotches. Its characteristics corresponded to the "embryoidogenic callus", which consists of round, small cells with dense cytoplasm and a large nucleus, which is characteristic of meristem cells (Figure 3). 
Table 2. Impact of Oryza sativa L. genotype on callusogenesis in isolated anthers culture, $\%$.

\begin{tabular}{|c|c|c|c|}
\hline \multirow{2}{*}{ № } & \multirow{2}{*}{ Sample № } & \multicolumn{2}{|c|}{ Callusogenesis, \% } \\
\hline & & $\min$ & $\max$ \\
\hline 1. & 04072 & 10,11 & 26,31 \\
\hline 2. & 02890 & 1,62 & 11,53 \\
\hline 3. & 0590 & 4,22 & 10,28 \\
\hline 4. & 01717 & 3,44 & 13,83 \\
\hline 5. & 04437 & 1,36 & 11,34 \\
\hline 6. & 04636 & 6,72 & 12,79 \\
\hline 7. & 04434 & 6,57 & 16,12 \\
\hline 8. & 04438 & 0,87 & 5,09 \\
\hline 9. & 04433 & 4,68 & 20,84 \\
\hline 10. & 04435 & 0,85 & 7,06 \\
\hline 11. & $98-4$ & 0,87 & 2,35 \\
\hline 12. & $04-89$ & 2,15 & 4,24 \\
\hline 13. & $70-05$ & 0,00 & 0,00 \\
\hline 14. & $265-08$ & 0,00 & 0,00 \\
\hline 15. & 111-09 & 0,08 & 0,23 \\
\hline 16. & 114-09 & 1,08 & 3,00 \\
\hline 17. & $208-09$ & 0,00 & 0,00 \\
\hline 18. & $58-12$ & 0,04 & 0,13 \\
\hline 19. & $1-14$ & 0,25 & 1,02 \\
\hline 20. & $2-14$ & 0,78 & 1,62 \\
\hline 21. & $3-14$ & 2,06 & 4,22 \\
\hline 22. & $8-14$ & 1,99 & 1,79 \\
\hline 23. & $11-14$ & 1,56 & 3,44 \\
\hline 24. & $25-14$ & 0,00 & 0,00 \\
\hline 25. & $68-14$ & 0,32 & 1,31 \\
\hline 26. & $71-14 \mathrm{M}$ & 4,62 & 9,54 \\
\hline 27. & $52-16$ & 0,54 & 1,46 \\
\hline 28. & Liaojing 168 & 3,21 & 11,85 \\
\hline 29. & Liaoxing 21 & 0,37 & 1,47 \\
\hline 30. & HY 11 & 1,94 & 3,64 \\
\hline
\end{tabular}

Table 3. Impact of Oryza sativa L. genotype on regeneration in isolated anthers culture, \%.

\begin{tabular}{|l|l|c|c|}
\hline \multirow{2}{*}{ № } & \multirow{2}{*}{ Sample № } & \multicolumn{2}{|c|}{ Regeneration, \% } \\
\cline { 3 - 4 } & & $\min$ & $\max$ \\
\hline 2. & 04072 & 1,62 & 7,82 \\
\hline 3. & 02890 & 0,33 & 3,51 \\
\hline 4. & 0590 & 0,50 & 3,54 \\
\hline 5. & 01717 & 0,84 & 3,04 \\
\hline 6. & 04437 & 1,03 & 1,47 \\
\hline 7. & 04636 & 0,94 & 5,73 \\
\hline 8. & 04434 & 0,68 & 8,12 \\
\hline 9. & 04438 & 0,31 & 2,73 \\
\hline 10. & 04433 & 1,15 & 4,70 \\
\hline 11. & 04435 & 0,12 & 3,52 \\
\hline 12. & $98-4$ & 0,00 & 0,00 \\
\hline 13. & $04-89$ & 0,00 & 0,00 \\
\hline
\end{tabular}




\begin{tabular}{|l|l|l|l|}
\hline \multirow{2}{*}{ № } & \multirow{2}{*}{ Sample № } & \multicolumn{2}{|c|}{ Regeneration, \% } \\
\cline { 3 - 4 } & & $\min$ & $\max$ \\
\hline 14. & $265-08$ & 0,00 & 0,00 \\
\hline 15. & $111-09$ & 0,00 & 0,00 \\
\hline 16. & $114-09$ & 0,00 & 0,00 \\
\hline 17. & $208-09$ & 0,00 & 0,00 \\
\hline 18. & $58-12$ & 0,00 & 0,00 \\
\hline 19. & $1-14$ & 0,00 & 0,00 \\
\hline 20. & $2-14$ & 0,00 & 0,00 \\
\hline 21. & $3-14$ & 0,00 & 0,00 \\
\hline 22. & $8-14$ & 0,55 & 0,97 \\
\hline 23. & $11-14$ & 0,00 & 0,00 \\
\hline 24. & $25-14$ & 0,00 & 0,00 \\
\hline 25. & $68-14$ & 0,00 & 0,00 \\
\hline 26. & $71-14 \mathrm{M}$ & 0,00 & 0,00 \\
\hline 27. & $52-16$ & 0,00 & 0,00 \\
\hline 28. & Liaojing 168 & 0,98 & 1,47 \\
\hline 29. & Liaoxing 21 & 0,07 & 0,33 \\
\hline 30. & HY 11 & 0,94 & 1,16 \\
\hline
\end{tabular}

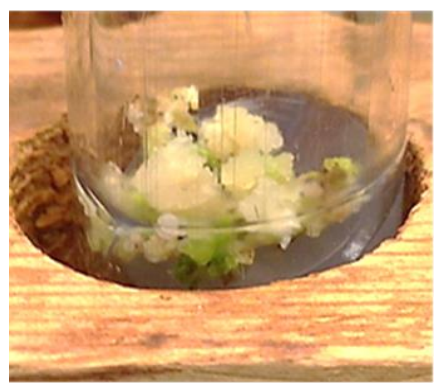

Fig. 3. Rice morphogenic callus.

With an increase in the cultivation time of pollen callus, its morphogenetic potential and ability to induce shoot formation decreased due to cell degradation due to progressive tissue necrosis. Therefore, to provoke morphogenetic potencies and increase the yield of green regenerants, callus was passaged on fresh nutrient media every 20-30 days. Viable rice regenerants from morphogenic types of callus were obtained from samples № 04072, 01717, 04437, 04433, 04636, 8-14 and Liaojing 168. Plants were formed according to the type of embryoidogenesis and gemmorizogenesis. In the embryoids / gemstones, coleoptile, shoot growth points with leaf rudiments and the meristem of the embryonic root were laid (Figure 4).

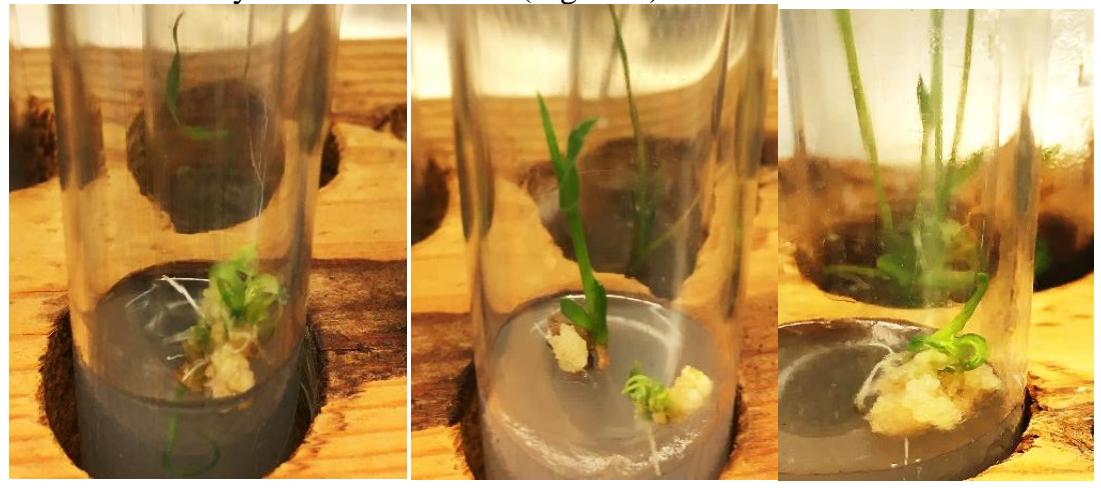

Fig. 4. Plants regeneration in rice anther culture in vitro. 
Identification of ploidy of regenerants obtained in anther culture was carried out for early detection of plants with a haploid set of chromosomes and timely transfer to the diploid level. A part of androgenic rice plants underwent spontaneous diploidization through endomitosis of haploid callus cells, as a result of which homozygous forms of samples № 01717, 04433, 04437 were obtained in the first year. Regenerants of samples № 04072, 8-14, Liaojing 168 and 04636 had a haploid set of chromosomes (12n), which was confirmed by cytological studies (Figure 5). These were sterile, undersized plants, with reduced sizes of vegetative and generative organs, and an increased number of spikelets per panicle. The regenerants were transferred to the dihaploid level using the embryonic panicle method.

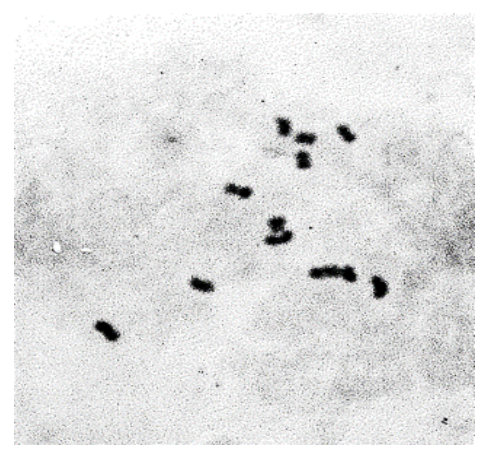

Fig. 5. Haploid set of chromosomes in rice roots (method of crushed preparations), 40x and 70x, water immersion.

Plants obtained through anther culture are rooted in liquid nutrient media and planted in vessels with soil for adaptation to ex vitro conditions. To check the genetic homogeneity of the obtained doubled haploids, DNA genotyping (microsatellite) analysis was performed using the profiles of 14 microsatellite DNA loci of two doubled haploids obtained on the basis of $\mathrm{F}_{1}$ hybrids (Table 4).

Table 4. Microsatellite profiles of rice samples obtained on the basis of doubled haploids.

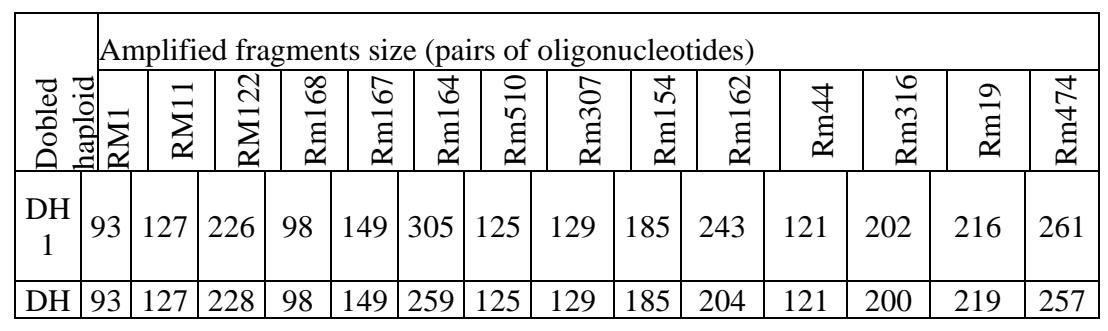

As a result of multiplex fragment analysis of doubled rice haploids for microsatellite markers RM1, RM11 and RM122, it was revealed that the genotypes obtained are homozygous in the studied DNA loci, which indicates their genetic homogeneity (Figure 6 in the interface of the GeneMapper 4.1 program window). 


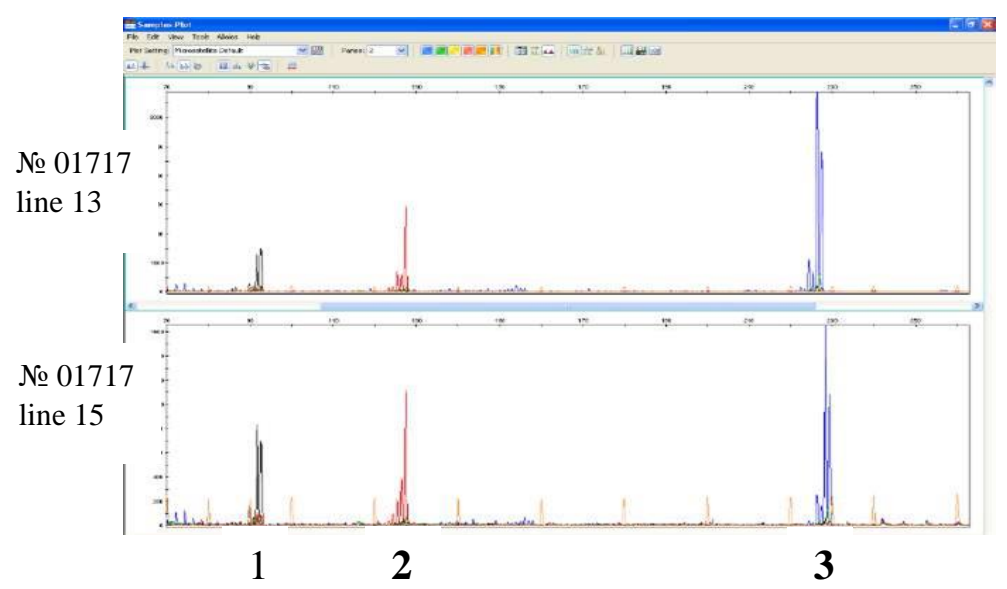

Fig. 6. The results of multiplex fragment analysis for microsatellite markers: 1-RM1, 2 -RM11 and 3 - RM122 of doubled haploids on the example of rice sample № 01711 lines 13 and 15.

\section{Discussion}

In the course of the experiments, from collection samples № 01717, 04433, 04437 carrying resistance genes Pi z, Pi 1, Pi 2, Pi 33, including 3 genes in one genotype (table), seeds of $30 \mathrm{DH}$ rice lines of three Russian genotypes were obtained, they were included in research on development of homozygous lines using the method of rice anther culture in vitro (Table $5)$.

Table 5. Characteristics of donor plants with blast resistance genes for development of DH lines in anther culture in vitro.

\begin{tabular}{|l|l|c|c|c|}
\hline $\begin{array}{c}\text { № in } \\
\text { catalogue }\end{array}$ & Sample name, gene & Variety type & $\begin{array}{c}\text { Growing period } \\
\text { till ripening, days }\end{array}$ & $\begin{array}{c}\text { Plant } \\
\text { height, } \\
\text { cm }\end{array}$ \\
\hline 01717 & No name $(\mathrm{Pi}-\mathrm{z})$ & italica & $105-115$ & $89-97$ \\
\hline 04433 & Б 33-38.6 (Pi-33) & italica & $108-112$ & $87-92$ \\
\hline 04437 & $\begin{array}{l}\text { Л-9 (Pi-1 + Pi-2 + Pi- } \\
\text { 33) }\end{array}$ & italica & $110-115$ & $90-96$ \\
\hline
\end{tabular}

Androgenic lines were propagated and evaluated by a complex of traits under the conditions of the growing experiment (Figure 7). Biometrics included plant height, bush shape, lodging and blast resistance, flag leaf angle, panicle shape, position, and length, number of spikelets per panicle, empty spikelets, empty grain, grain index $(1 / b)$, grain weight per panicle, and weight of 1000 grains. 


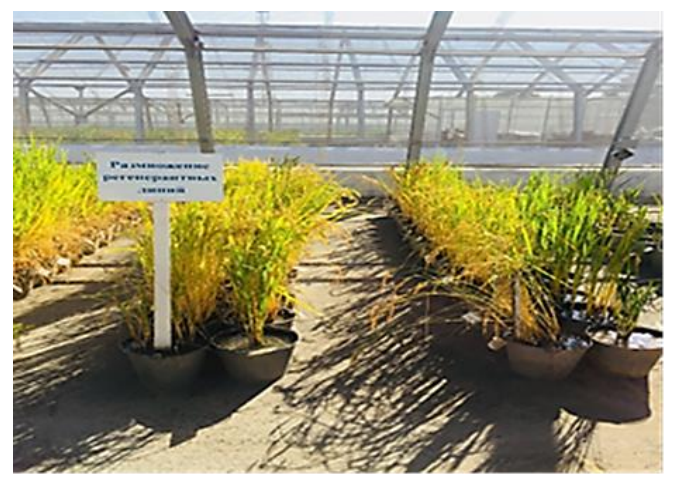

Fig. 7. Propagation and evaluation of DH lines developed from rice samples with blast resistance genes.

Analysis of the variability of traits of $\mathrm{DH}$ androgenic lines showed that in the populations of dihaploids, a wide range of variation in vegetative, morphological, and biological traits was observed. This was a regularity for DH populations of all genotypes. So, for example, in population № 01717 of 18 homozygous lines, differences in plant height were noted: from 75-80 cm for line № 3 to $115-126 \mathrm{~cm}$ for line № 18 . Line № 3 belongs to the group of low-growing plants - $75.0-80.0 \mathrm{~cm}$, which is $14-17 \mathrm{~cm}$ lower than that of the donor line. DH-lines № 11, 13-16, having a height at the level of the parental forms, are assigned to the group of medium-sized. Androgenic lines №1-2, 4-10, $12,17-18$ had plant heights above $100 \mathrm{~cm}$, which significantly exceeded the height of the original forms by $11-26 \mathrm{~cm}$. These lines were classified as tall.

Out of 18 lines, 17 had a medium-spreading drooping panicle, and only one line № 3 had a vertical compact one. Differences in traits (elements of productivity) were noted: the plants had a panicle length from $14.0 \mathrm{~cm}$ in line №14 to $21.5 \mathrm{~cm}$ in line № 18, with a significant difference in the number of spikelets from 41.0 pcs. at line № 14 up to 155.4 pcs. for line № $18\left(\mathrm{LSD}_{05}=1.5\right)$, while the mass of 1000 grains was the same $-26.5 \mathrm{~g}$ and $26.6 \mathrm{~g}\left(\mathrm{LSD}_{05}=1.0\right)$, respectively. All lines had a low empty grain content from 11.5 to $35.8 \%$, except for line № $3-46.6 \%$ (average).

The location of the flag leaf in the samples varied from 15 to 60 degrees. Lines №№ 2, 15,17 and 18 showed themselves as resistant (3 points) to blast, the rest as highly resistant (1 point) (table 6$)$.

The mass of 1000 grains is an important economic trait that characterizes the quality of the seed material. It is related to the size and filling of the seeds. The mass of 1000 grains of lines № 4-6, 9-14, 16-18 of sample 01717 was average (26.2-29.1 g). The variability for this trait of all lines ranged from 22.0 to 29.1 grams (Table 7).

All lines № 04433 B 33-38.6 had a height at the level of donor plants. The number of spikelets on a panicle between lines varied from 90.4 to 127.8 pcs. ( $\operatorname{LSD}_{05}=14.8$ ). Lines № 1 and 5 had a high empty grain content (73.0 and 69.8\%, respectively). Lines 4 and 5 had a weakly spreading vertical panicle, 1-3 lines - a vertical compact one. Lines № 1, 3 and 4 proved to be resistant to blast, lines № 2 and 5 as highly resistant.

Seven lines 04437 L.9 had a height significantly lower than the donor plants; in terms of plant height they were classified as low-growing $(60-79 \mathrm{~cm})$, while the donor forms had an average height $(90-96 \mathrm{~cm})\left(\mathrm{LSD}_{05}=12.7\right)$. Lines № 1,4 and 5 showed themselves as highly resistant to blast, lines № 2, 6 and 7 as resistant, line №3 had an intermediate character. All lines had an upright compact panicle. The location of the flag leaf in the samples varied from 25 to 45 degrees (Table 6). The number of spikelets on panicles of lines of this sample varied from 84.0 pcs. up to 109.2 , and empty grain from 21.6 to $55.8 \%$ (table 7). 
Table 6. Characteristics of DH lines developed from samples with blast resistance genes for economic and biological traits, 2020

\begin{tabular}{|c|c|c|c|c|c|c|c|c|}
\hline 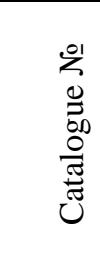 & 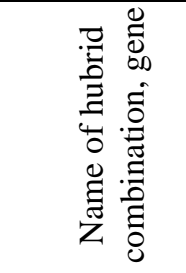 & 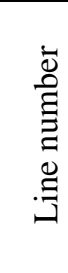 & 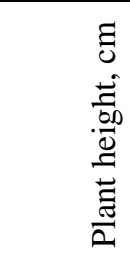 & 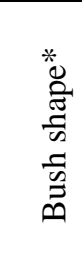 & 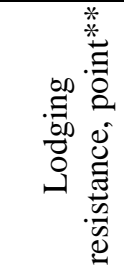 & 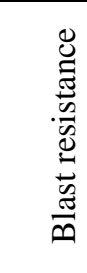 & 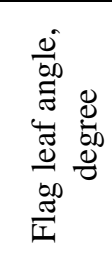 & 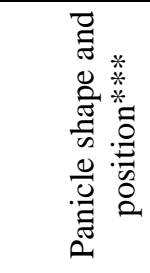 \\
\hline \multirow[t]{18}{*}{01717} & \multirow{18}{*}{$\begin{array}{l}\text { no name, } \\
\text { Pi-z }\end{array}$} & 1 & $110-115$ & 1 & 1 & 1 & 80 & $5 / 9$ \\
\hline & & 2 & $105-112$ & 1 & 1 & 3 & 45 & $5 / 9$ \\
\hline & & 3 & $75-80$ & 1 & 1 & 1 & 15 & $1 / 1$ \\
\hline & & 4 & $110-117$ & 1 & 1 & 1 & 50 & $5 / 9$ \\
\hline & & 5 & $110-113$ & 1 & 1 & 1 & 45 & $5 / 9$ \\
\hline & & 6 & $100-110$ & 1 & 1 & 1 & 35 & $5 / 9$ \\
\hline & & 7 & $108-112$ & 1 & 1 & 1 & 45 & $5 / 9$ \\
\hline & & 8 & $110-113$ & 1 & 1 & 1 & 35 & $5 / 9$ \\
\hline & & 9 & 110-119 & 1 & 1 & 1 & 45 & $5 / 9$ \\
\hline & & 10 & $100-105$ & 1 & 1 & 1 & 15 & $5 / 9$ \\
\hline & & 11 & $97-104$ & 1 & 1 & 1 & 15 & $5 / 9$ \\
\hline & & 12 & $110-117$ & 1 & 1 & 1 & 45 & $5 / 9$ \\
\hline & & 13 & $107-110$ & 1 & 1 & 1 & 35 & $5 / 9$ \\
\hline & & 14 & $95-106$ & 1 & 1 & 1 & 15 & $5 / 9$ \\
\hline & & 15 & $97-110$ & 1 & 1 & 3 & 40 & $5 / 9$ \\
\hline & & 16 & $95-102$ & 1 & 1 & 1 & 50 & $5 / 9$ \\
\hline & & 17 & $105-117$ & 1 & 1 & 3 & 60 & $5 / 9$ \\
\hline & & 18 & $115-126$ & 1 & 1 & 3 & 50 & $5 / 9$ \\
\hline \multirow[t]{5}{*}{04433} & \multirow{5}{*}{$\begin{array}{l}\text { B 33-38,6 } \\
\text { Pi-33 }\end{array}$} & 1 & $90-95$ & 1 & 3 & 1 & 35 & $1 / 1$ \\
\hline & & 2 & $83-87$ & 1 & 1 & 1 & 30 & $1 / 1$ \\
\hline & & 3 & $75-80$ & 1 & 3 & 1 & 35 & $1 / 1$ \\
\hline & & 4 & $80-86$ & 1 & 3 & 3 & 35 & $3 / 1$ \\
\hline & & 5 & $78-88$ & 1 & 1 & 1 & 45 & $3 / 1$ \\
\hline \multirow[t]{7}{*}{04437} & \multirow{7}{*}{$\begin{array}{l}\text { L.9 } \\
\text { Pi-1 + Pi-2 + } \\
\text { Pi-33 }\end{array}$} & 1 & $75-861$ & 1 & 1 & 1 & 35 & $1 / 1$ \\
\hline & & 2 & $75-84$ & 1 & 1 & 3 & 35 & $1 / 1$ \\
\hline & & 3 & 60-67 & 1 & 1 & $1-3$ & 45 & $1 / 1$ \\
\hline & & 4 & $60-65$ & 1 & 3 & 1 & 45 & $1 / 1$ \\
\hline & & 5 & $65-74$ & 1 & 3 & 1 & 25 & $1 / 1$ \\
\hline & & 6 & $65-70$ & 1 & 3 & 3 & 35 & $1 / 1$ \\
\hline & & 7 & $65-79$ & 1 & 1 & 3 & 45 & $1 / 1$ \\
\hline
\end{tabular}

Note: * - erect; $* *_{-} 1$ point - resistant; 3 points - medium resistant;

*** - 1/1 - vertical compact; 5/9 - medium-spreading drooping; $3 / 1$ - weakly-spreading vertical;blast resistance 1- highly resistant; 3 - resistant

Analysis of the variability of traits of the obtained androgenic lines showed that the gametoclonal variability of regenerated plants in anther culture in vitro significantly exceeds the variability of regenerated plants obtained in somatic cell culture (somaclonal variability), since the main mechanism of its occurrence is not the mutation process, but gene recombination in meiosis with the formation of male reproductive gametes. Variability was noted for such traits as plant height, panicle length, blast resistance, 1000 
grain weight. The grain size of most of the samples under study was at the average level, the variation in the mass of 1000 grains along the lines studied was from 17.1 to 29.1 grams. Depending on the genotype, the range of variability of the trait "grain weight per panicle" in the studied samples ranged from 0.9 to $2.8 \mathrm{~g}$. The indicator of the "panicle length" trait varied in the range of $12.3-21.5 \mathrm{~cm}$. The variability of empty grain in the studied genotypes was in the range of $11.5-57.3 \%$. In terms of the number of spikelets formed on a panicle, lines №18 of sample 01717/16 (155.4 pcs.) and № 1 and 5 of sample 04433/18 (123.0 and 127.8 pcs., respectively) had an advantage. The bush shape is erect for all lines. All DH lines obtained by the method of experimental haploidy showed themselves to be highly resistant and resistant to blast. There were no lines with signs of blast disease.

Table 7. Productivity elements of DH-lines panicles, 2020

\begin{tabular}{|c|c|c|c|c|c|c|c|c|}
\hline 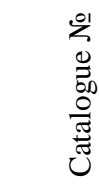 & 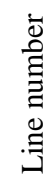 & 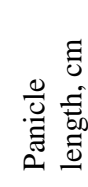 & 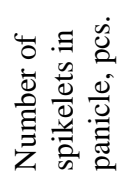 & 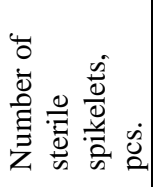 & 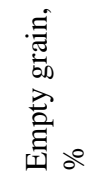 & $\begin{array}{l}\text { Grain } \\
\text { index } \\
(1 / b)\end{array}$ & 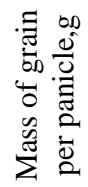 & 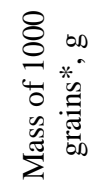 \\
\hline \multirow[t]{18}{*}{01717} & 1 & 16,5 & 103,0 & 24,8 & 24,1 & 2,7 & 1,9 & 25,4 \\
\hline & 2 & 18,7 & 108,6 & 27,8 & 25,6 & 2,8 & 2,2 & 25,5 \\
\hline & 3 & 17,9 & 74,2 & 34,6 & 46,6 & 2,3 & 0,9 & 22,3 \\
\hline & 4 & $\begin{array}{ll}18,0 \\
\end{array}$ & 88,6 & 11,2 & 12,6 & 2,5 & 2,2 & 29,1 \\
\hline & 5 & 15,4 & 60,6 & 7,0 & 11,5 & 2,3 & 1,5 & 26,9 \\
\hline & 6 & 15,3 & 59,6 & 12,8 & 21,5 & 2,3 & 1,3 & 26,7 \\
\hline & 7 & 16,7 & 72,0 & 13,4 & 18,6 & 2,5 & 1,6 & 25,5 \\
\hline & 8 & 17,8 & 91,6 & 32,8 & 35,8 & 2,5 & 1,6 & 26,4 \\
\hline & 9 & 17,6 & 79,0 & 19,6 & 24,8 & 2,6 & 1,6 & 26,7 \\
\hline & 10 & 15,3 & 47,4 & 9,6 & 20,3 & 2,7 & 1,1 & 26,5 \\
\hline & 11 & 15,8 & 59,2 & 7,4 & 12,5 & 2,8 & 1,4 & 27,5 \\
\hline & 12 & 15,4 & 63,8 & 9,0 & 14,1 & 2,4 & 1,5 & 27,2 \\
\hline & 13 & 15,1 & 55,6 & 10,6 & 19,1 & 2,9 & 1,2 & 26,5 \\
\hline & 14 & 14,0 & 41,0 & 7,6 & 18,5 & 2,6 & 1,0 & 26,5 \\
\hline & 15 & 14,9 & 59,8 & 20,2 & 33,8 & 2,8 & 1,1 & 24,8 \\
\hline & 16 & 15,6 & 62,8 & 9,2 & 14,6 & 2,8 & 1,4 & 27,5 \\
\hline & 17 & 14,7 & 50,0 & 12,2 & 24,4 & 2,8 & 1,3 & 26,2 \\
\hline & 18 & 21,5 & 155,4 & 36,8 & 23,7 & 2,7 & 2,8 & 26,6 \\
\hline \multirow[t]{5}{*}{04433} & 1 & 17,8 & 123,0 & 73,0 & 59,3 & 1,7 & 1,1 & 20,8 \\
\hline & 2 & 16,0 & 90,4 & 36,6 & 40,5 & 1,8 & 1,2 & 20,7 \\
\hline & 3 & 17,5 & 100,2 & 34,2 & 34,1 & 1,8 & 1,4 & 20,1 \\
\hline & 4 & 17,1 & 107,6 & 36,6 & 34,0 & 1,7 & 1,3 & 20,8 \\
\hline & 5 & 17,2 & 127,8 & 69,8 & 54,6 & 1,8 & 1,8 & 20,9 \\
\hline \multirow[t]{7}{*}{04437} & 1 & 15,1 & 95,2 & 27,0 & 28,4 & 1,8 & 1,3 & 19,1 \\
\hline & 2 & 14,5 & 92,8 & 21,6 & 23,3 & 1,8 & 1,4 & 19,6 \\
\hline & 3 & 13,5 & 90,8 & 52,0 & 57,3 & 1,8 & 0,8 & 23,2 \\
\hline & 4 & 12,3 & 84,0 & 44,8 & 53,8 & 2,0 & 0,8 & 19,2 \\
\hline & 5 & 14,7 & 112,2 & 49,2 & 43,8 & 2,0 & 1,2 & 19,0 \\
\hline & 6 & 13,9 & 109,2 & 55,8 & 51,1 & 1,8 & 1,9 & 17,1 \\
\hline & 7 & 14,6 & 106,2 & 44,0 & 41,4 & 2,0 & 1,2 & 19,0 \\
\hline \multicolumn{2}{|c|}{ LSD $_{005}$} & 1,50 & 14,8 & & & & 0,5 & 1,0 \\
\hline
\end{tabular}

Note -* mass of 1000 grain at $14 \%$ moisture.

From 30 DH, 5 promising lines of three genotypes were selected: 01717 - lines № 4, 9, 14, 15; 04437 - line № 7 with a complex of economically valuable traits and specified properties. They were transferred for study in breeding and then control nurseries. The best samples of the control nursery as candidates for varieties will become participants in a competitive variety testing, for the formation of which the yield, growing season, plant 
height, panicle weight, spikelet pubescence, field assessments of resistance to lodging, diseases, shedding, and varietal uniformity will be carried out.

\section{Conclusions}

The variability in callusogenesis / regeneration traits was studied, on the basis of which rice genotypes responsive to anther culture were identified. Accelerated by the method of experimental haploidy on the basis of spontaneous diploidization in vitro from sources with genes for resistance to blast disease, a new genetic source material with high morphological and genetic uniformity was developed. Progeny of the $\mathrm{R}_{2}$ generation regenerants, possessing a wide variety of traits, were obtained and evaluated. Based on the analysis of plants in populations of doubled haploids, a wide range of variation in traits (productivity elements) was noted. Seed offspring of these samples were transferred to the USU "Collection of genetic resources of rice, vegetables and melons" to replenish the collection of doubled haploids and for study in a breeding nursery. The results obtained during the work can be used for further development of research in the field of artificial androgenesis.

\section{References}

1. Dwivedi S L, Britt A B, Tripathi L, Sharma S, Upadhyay H D, Ortiz R 2015 https://doi.org/10.1016/j.biotechadv.2015.07.001 33 812-29

2. Wedzony M, Foster B P, Zur I, Golemiec E, Szechynska-Hebda M, Dubas E, Gotebiowska G 2009 SpringerScience + BusinessMedia 5 1-35

3. Germana M A 2011 Plant Cell Tiss Org Cult. 104 283-300

4. Weyen J, Touraev A, Foster B P, Jain E M 2009 SpringerScience + BusinessMedia 5 179-89

5. Urazaliev KR, Orsini KhM, Abekova AM, Bazylova TA, Daniyarova AK 2013 Bulletin of KazNU Series: ecological 2/2 (38) 369-74

6. Ayed O S, Buyser J, Picard E, Trifa Y, Amara H S 2010 J. Plant Breed. Crop. Sci. 2 $30-8$

7. AbdRahman Z, AhmadSeman Z,' NazreenaOthman A,' BahagiaAb M, Shahril G et al' 2021 Biocatalysis and Agricultural Biotechnology $31 \quad 101865$ https://doi.org/10.1016/j.bcab.2020.101865

8. Orłowska R, Pachota K, Machczyńska J, Niedziela A, Makowska K, Zimny J, $\begin{array}{lllllll}\text { Bednarek P } 2020 \text { Electronic Journal of Biotechnology } & \mathbf{4 3} & 8-15\end{array}$ https://doi.org/10.1016/j.ejbt.2019.11.001

9. Savenko E, Mukhina Zh, Glazyrina V, Korotenko T, Garkusha S 2019 IOP Conference Series: Earth and Environmental Science 3110186 doi: 10.1088/17551315/403/1/01

10. Mishra R, Jwala G, Rao N 2016 Rice Science 23 57-68 https://doi.org/10.1016/j.rsci.2016.02.001

11. Bernardo R 2009 Theor. Appl. Genet. 119 255-62

12. Zheng M Y 2003 Plant Cell Tiss Org Cult. 73 213-30

13. Belicuas P R, Guimarães C T, Paiva L V, Duarte J M, Maluf W R, Paiva E Euphytica 156 (2007) 95-102 